\title{
Behavioral Medicine Methods in Treatment of Somatic Conditions
}

\author{
Joanna Białkowska $\mathbb{D}^{1},{ }^{1}$ Jakub Juranek $\mathbb{D},^{2}$ and Joanna Wojtkiewicz $\mathbb{D}^{3}$ \\ ${ }^{1}$ Department of Psychology, Sociology of Health and Public Health. School of Public Health, Collegium Medicum, University of \\ Warmia and Mazury, Clinical University Hospital in Olsztyn, 10-900 Olsztyn, Poland \\ ${ }^{2}$ Institute of Psychology, Polish Academy of Sciences, Jaracza 1, 00-378 Warsaw, Poland \\ ${ }^{3}$ Department of Pathophysiology, School of Medicine, Collegium Medicum, University of Warmia and Mazury, \\ 10-900 Olsztyn, Poland
}

Correspondence should be addressed to Joanna Wojtkiewicz; joanna.wojtkiewicz@uwm.edu.pl

Received 12 May 2020; Revised 30 August 2020; Accepted 27 October 2020; Published 3 November 2020

Academic Editor: Diane Ruge

Copyright (c) 2020 Joanna Białkowska et al. This is an open access article distributed under the Creative Commons Attribution License, which permits unrestricted use, distribution, and reproduction in any medium, provided the original work is properly cited.

\begin{abstract}
Background. The aim of this article is to present a short review of noninvasive, nonpharmacological treatment methods used in somatic illnesses that fall under the umbrella of approach called behavioral medicine. Methods. The narrative review method was applied in the study. Science paper databases, including PubMed, had been used to retrieve papers on therapeutic methods used in clinical setting that meet the broad criteria of behavioral medicine definition as stated in the Charter of International Behavioral Medicine Society Results. Main groups of methods, disorders in which they are being employed and their effectiveness, have been identified. Conclusions. Behavioral medicine is grouping treatment methods and interventions that hold large potential for clinical setting. Two groups of methods can be distinguished by the scrutiny and level of evidence gathered in their effectiveness assessment; for biofeedback, guided imagery, and hypnosis techniques, comprehensive evidence reports in the framework of U.S. Evidence Synthesis Program exist. Meditation techniques, disclosure therapies, and relaxation methods are less well assessed. Broader employment of behavioral medicine therapies in clinical setting is possible after addressing two major problems in the field, which are deficiencies in quality evidence of effectiveness for many of the methods and their insufficiencies in underlying therapeutic mechanism knowledge.
\end{abstract}

\section{Introduction}

Since before the emergence of modern medicine, it has been observed that behavior and states of mind have relation with health and illness. This important understanding, broadly present in ideas of ancient thinkers like Aristotle, Galen, and Islamic Golden Age Persian physicians like Abu Zayd Al-Balkhi and 'Ali b. al-'Abbas al-Majusi (latinized: Haly Abbas), had been somewhat subsided over time with the successful raise of mechanistic paradigm in medicine that followed works of academics such as William Harvey and his explanation of heart functioning as a pump or Rene Descartes notion of mind and body as utterly separate "machineries" $[1,2]$. As late as the first half of $20^{\text {th }}$ century, the reemergence of awareness that crucial links exist between behavior, mental processes, and body functioning was witnessed. With rise of behaviorism and works of figures such as Ivan Pavlov, previously undescribed connections, like between central nervous and digestive system, mediated by conditioning, became exposed. Accumulation of such knowledge leads to eventual advent of psychosomatic medicine [3] and later distinct from its forebearer-behavioral medicine $[4,5]$.

Behavioral medicine is interdisciplinary field, integrating and using psychosocial, behavioral, and biomedical knowledge relevant to health and illness, in an effort to understand fundamental biobehavioral mechanisms of well-being and serve the needs of clinical diagnosis, intervention, disease prevention, and health promotion [6-8]. It incorporates conceptual meanings of the term "behavioral" on four distinctive levels, as elucidated by Weiss and Schwartz [5, 9]. At the primary level, "behavioral" means relation with concepts and techniques derived from behavioral therapy, as the behavioral medicine field was first inspired by advances in operant 
conditioning and the emergence of biofeedback theory. The second level pertains broader health relevant concepts and techniques, which are subsets of wider, more general psychological knowledge, like ideas originating from positive psychology and findings coming from affiliated discipline of health psychology. The third level is "behavior" conceptualized also in wider context of social sciences like sociology or anthropology, enabling appreciation of variables like social support or spirituality in context of health. The fourth level translates "behavioral" as functional, meaning interest in common, high-level principles that apply to the functioning of different systems at various levels of reality, keeping an open door for potentially usable and significant wisdom coming from far related, or unrelated, fields, spanning from physics to ecology.

The interests and interdisciplinary character of behavioral medicine is responsible for its strong interrelation with other health oriented disciplines and subdisciplines that have rooting in psychology, namely, psychoneuroimmunology [10], psychoendocrinology [11], psychooncology [12], and the broadest, earlier mentioned, psychology of health [13]. A common trait of all these health-related fields is emphasis on holistic bio-psycho-social (or-as extended by recent propositions-bio-psycho-socio-cultural [13]) approach to health [14].

Due to its interdisciplinary aspect, the essence of combining knowledge from broad spectrum of areas with the aim to facilitate synergy, blending skills and ideas, behavioral medicine has proved to be currently, and ventures to be in the future, a fertile ground for deriving useful and innovative strategies for the prevention, diagnosis, and treatment of a wide range of health problems. Much potential is being recognized in this field in terms of new treatments and improving cost-effectiveness of health care systems [15].

Realization of behavioral medicine potential depends on proper research and availability of information regarding existing solutions in that framework. The aim of this paper is to review contemporarily applied and researched interventions used in the treatment of somatic conditions, falling into the domain of behavioral medicine by its definition. Although formal birth of this discipline had taken place just in the year 1977 (with the founding Yale Conference on Behavioral Medicine [16]), treatments included in this review often have longer history. The inclusion criterion in this paper was given treatment matching the scope of behavioral medicine definition, as outlined in the Charter of International Behavioral Medicine Society [7], and not its history or theoretical origin.

\section{Review of Therapeutic Method Groups}

Interventions falling into behavioral medicine category are primarily nonpharmacological techniques, originating from the appreciation of the interrelations between the body and mind. Many of them are based on the employment of principles of learning (conditioning), or recognition of the importance stress (of various origins) has on organism (including its significant impact on functioning of the immune system). Treatment approaches include a group of biofeedback methods, relaxation techniques (also biofeedback supported relaxation), disclosure interventions, hypnosis, guided imagery and visualization techniques, meditation, and bulk, stress-management programs. Some of the methods amalgamate and share common features, benefiting from nonidiosyncratic mechanisms; for example, types of meditation can include focus on briefing, visualizations, and relaxation, and several of the methods possess common aspect of being founded on principles of learning. Many of the separate techniques are often combined and administered under a joint umbrella of stress-management programs [17-24].

Two separate groups of methods can be distinguished by the scrutiny and level of evidence gathered in their effectiveness assessment; for biofeedback, guided imagery, and hypnosis techniques, comprehensive evidence reports in the framework of U.S. Evidence Synthesis Program exist, and so unified style summarizing tables depicting condition and outcome area effectiveness are provided. The remaining methods' effectiveness and area of application are reported in the text or different form of tables.

\section{Biofeedback}

Associated with the founding roots of behavioral medicine movement in science and connected with the first sense of term "behavioral"-meaning related to behavioral therapy and methods-biofeedback techniques rely primarily on the principles of operant conditioning. The essence of the wide group of these interventions is learning by individual how to voluntarily control body processes-which are commonly falsely considered involuntary-for health benefits or symptom control. That is why, the term "treatment," emphasizing the curing goal of these methods, but accentuating passivity, is better replaced here with the word "training," highlighting the need of active patient participation in the learning and thus curing process. Biofeedback methods substantially employ equipment that converts physiological input from the body into auditory or visual signals that constitute the feedback for the patient. With such feedback, a person can clearly learn how their mental states are resembled in the physiological state of the system targeted in particular biofeedback intervention and try modifying and managing it, typically with support of a qualified practitioner. Instrumentation used in different biofeedback methods measures muscle activity, skin temperature, electrodermal activity (sweat gland activity), respiration, heart rate variability, blood pressure, brain electrical activity and blood flow [25]. Biofeedback techniques are employed in two modes: alone (less often) or combined with stress management, relaxation, psychotherapeutic and other techniques [17].

One of the most common biofeedback trainings involves electromyography (EMG), conveying feedback regarding muscle activation, the intensity of muscle contraction, and manifestation of muscle fatigue [26]. Electromyography feedback in clinical setting has been pioneered in the 1920 s by Edmond Jacobson [27-29]. It has been tried since, to treat various conditions, among which are arthritis [30], asthma [31], Bell's palsy [32], cerebral palsy [33, 34], hand dystonia $[35,36]$, repetitive strain injury [37], and spasmodic 
torticollis [38]. It has proved to be the most efficacious in the treatment of various kinds of chronic pain [25], headaches (tension, migraine, or of mixed origin) $[39,40]$, temporomandibular disorder [41], and constipation [42].

Another biofeedback method is thermal biofeedback. It is associated with self-regulation of skin temperature [43]. This type of biofeedback is usually performed with the use of skin temperature sensors, called thermistors, attached to fingers, with visual or tonal feedback to the subjects. Thermal biofeedback is usually administered with other modalities of biofeedback techniques and demonstrated to be useful in the treatment of Raynaud's disease [43], headaches [44], and chronic pain conditions $[25,45]$.

The idea behind yet another biofeedback mode-skin conductance feedback-is to deliver person information about sweat gland activity, which is closely correlated with sympathetic nervous system functioning [46]. With very short response time falling in the range of below two seconds, skin conductance activity (SCA) feedback-known also as EDA (electrodermal activity) or GSR (galvanic skin response) feedback-provides superb sensitivity to emotional changes [25]. This biofeedback technique receives a lot of attention as a complementary epilepsy treatment with encouraging outcomes reported regarding its efficiency [47-50]. Other medical uses have been and are being studied, including employment of this technique in support of controlling glucose levels in type 2 diabetes [51], treatment of Tourette syndrome [52], and headaches [53].

Another example of biofeedback technique is heartrate variability (HRV) feedback. It involves monitoring either heart rate alone or heart rate plus respiration. Data regarding heart rate is collected via electrocardiograph (EKG) monitors or plethysmographic sensors on the finger or earlobe [25]. It is has been tried and used in a variety of disorders, with multiple potential pathways for its therapeutic effects proposed $[54,55]$. Best results were reported in treating recurrent abdominal pain [56], chronic muscle pain [57], and cyclic vomiting [58].

Arguably, the most popular biofeedback application could be electroencephalogram (EEG) neurofeedback (EEG-NFB) - a method which takes advantage of the fact that particular brain wave patterns correlate with specific disorders and syndromes [59]. Improvement in functioning in these disorders may be possible due to the changes in the amplitudes or overall proportion of brain wave types. These changes occur as a result of teaching the patient to strengthen the desired brain wave frequencies, amplitudes, or potentials and inhibit the undesirable ones. There are three main protocols used in EEG-NFB interventions. The most commonly used is the frequency training, in which the goal is changing the power ratio of the EEG frequency bands. It is mostly used in attention deficit hyperactivity disorder (ADHD) and autism spectrum disorders treatment $[60,61]$. Slow cortical potential (SCP) is a second kind of popular neurofeedback protocol, based on modulating specific (slow) event-related potentials (negative or positive); it is also mainly used in ADHD. In somatic disorders, a third protocol is however most widely used. It is coherence training. The aim of this method is promoting the coherence of activity (frequency as well as amplitude and phase) of different brain regions. Theoretical foundation for this protocol lay in the fundamental observation that specific decoherence (or distorted connectivity) often accompanies particular disorders.

EEG neurofeedback is widely used to treat epilepsy [62]; it is also being administered in novel capacities to treat conditions such as fibromyalgia [63] or chronic prostatitis [64] and is commonly adopted in rehabilitation, for example, in patients with amyotrophic lateral sclerosis (ALS) [65], stroke [66], spinal cord [67], and traumatic brain injuries [68, 69].

Doubts about utility of neurofeedback methods exist and stem mainly from three reasons, as comprehensively outlined by Thibault and Raz [70]. One reason is the deficiency of methodologically high-level, large, quality-controlled, and randomized studies proving its effectiveness in vast amount of conditions it is applied to. Another cause is existence of the so-called sham neurofeedback effects: placebo-like, positive results, obtained using feedback taken from the activity of other than the trained person brain or similarly irrelevant source [71]. The third reason is lack of widely acknowledged and established mechanisms that would explain the claimed therapeutic benefits. The research work addressing these problems is however already being conducted, with good outlook regarding accumulation of better evidence considering effectiveness and establishment of the psychological and neural mechanisms underlying clinical benefits [72].

Current EEG neurofeedback techniques serve as an inspiration for developing more advanced brain-subject feedback loops, employing the functional Magnetic Resonance Imaging (fMRI) systems. This relatively new (emerging in year 2003) biofeedback expanse area is still mostly field of ongoing research with potential yet to be evaluated, less a ground of mature clinical applications [73-75]. The surge of interest in this subject has been reported in the last decade, and promising new technical advances and protocols are in development. Examples of these are the decoded neurofeedback (DecNef) technique-aiming at inducing a specific activity pattern within a target brain region-and functional connectivity-based neurofeedback (FCNef), in which the goal is changing functional connectivity between different regions [74].

In the beginning of the $21^{\text {st }}$ century, the Task Force of the Association for Applied Psychophysiology and Biofeedback plus the Society for Neuronal Regulation formulated criteria for designated levels of evidence-based clinical efficacy of psychophysiological interventions. They span from level 1-not empirically supported-to level 5-efficacious and specific [76]. According to Yucha and Montgomery [25], who rated evidence of efficacy in conformance with the abovementioned criteria in 2008, there were eleven (see Table 1) conditions for which biofeedback training could be considered either as "efficacious and specific" or as "efficacious" (level 5 and level 4) and further nine conditions in which it was rated as being probably efficacious (these include arthritis, diabetes mellitus, and urinary incontinence in males), with additional eighteen were possibly efficacious (level 2). The one condition which fits into the "efficacious and specific" level is urinary incontinence in females. General effectiveness (level 4) has been stated for anxiety, attention 
TABLE 1: Medical conditions for which evidence suggests biofeedback therapies are "efficacious and specific" (level 5) and "efficacious" (level 4) according to a work by Yucha and Montgomery [25]. Types of biofeedback therapy approaches are listed next to each condition.

\begin{tabular}{|c|c|c|}
\hline $\begin{array}{l}\text { Efficacy evidence level } \\
\text { 5-efficacious and specific }\end{array}$ & Condition & Method \\
\hline \multirow{11}{*}{$\begin{array}{l}\text { Efficacy evidence level } \\
4 \text {-efficacious }\end{array}$} & $\begin{array}{l}\text { Urinary incontinence } \\
\text { in females }\end{array}$ & Pelvic floor muscles biofeedback \\
\hline & Anxiety & Biofeedback (various modalities, including EMG and EEG) \\
\hline & $\begin{array}{c}\text { Attention deficit } \\
\text { hyperactivity disorder }\end{array}$ & EEG biofeedback \\
\hline & Chronic pain & $\begin{array}{c}\text { Biofeedback (EMG, thermal \& EEG biofeedback-utilization for given disorder } \\
\text { best determined by consulting literature for that specific condition) }\end{array}$ \\
\hline & Constipation in adults & EMG and manometry biofeedback \\
\hline & Epilepsy & EEG biofeedback \\
\hline & Headache in adults & EMG biofeedback \\
\hline & Hypertension & $\begin{array}{c}\text { Thermal, electrodermal response (EDR), heart rate, EMG, or direct blood pressure } \\
\text { biofeedback }\end{array}$ \\
\hline & Motion sickness & Galvanic skin response (GSR) biofeedback \\
\hline & Raynaud's disease & Thermal biofeedback \\
\hline & $\begin{array}{l}\text { Temporomandibular } \\
\text { disorder }\end{array}$ & EMG biofeedback \\
\hline
\end{tabular}

TABLE 2: Health conditions for which biofeedback interventions had evidence of a positive effect on diagnosis-related, secondary, or global outcomes according to the U.S. Department of Veterans Affairs Evidence Synthesis Program (ESP) report published in 2019 [77].

\begin{tabular}{lccc}
\hline Condition & Diagnosis-related outcomes & Secondary outcomes & Global outcomes \\
\hline Fecal incontinence & $\mathrm{X}$ & $\mathrm{X}$ & \\
Headache & $\mathrm{X}$ & & $\mathrm{X}$ \\
Stroke & $\mathrm{X}$ & & \\
Urinary incontinence after prostatectomy & $\mathrm{X}$ & & $\mathrm{X}$ \\
\hline
\end{tabular}

deficit hyperactivity disorder (ADHD), chronic pain, constipation in adults, epilepsy, headache in adults, hypertension, motion sickness, Raynaud's disease, and temporomandibular disorder (TMD).

More recent report compiled under the auspices of the US Veterans Health Administration Evidence Synthesis Program (EVP) [77, 78] concluded (see Table 2) there is strong, high-confidence evidence for biofeedback intervention efficacy in clinical conditions like pain resulting from migraine or tension headaches and urinary incontinence in men after a prostatectomy. Moderate evidence was found supporting beneficial effects in fecal incontinence and stroke treatment. Contrary to summary by Yucha and Montgomery [25], hypertension, woman urinary incontinence outcomes for fibromyalgia, was described as providing no benefits, but with low-confidence evidence base. For the vast majority of other biofeedback clinical applications, the authors of the report abstained from conclusions stating insufficient data.

\section{Hypnosis}

Hypnosis is a method of inducing in an individual a state of hypnotic trance, a mode of deep relaxation and focus with increased suggestibility and suspension of critical faculties. In this special state of consciousness, two types of suggestions can be made to the affected person: direct hypnotic suggestion, made while the person is in trance, which alters behavior or perception while the trance persists, and posthypnotic suggestion that alters behavior or perception after the trance ends. Typical hypnotherapy consists of hourly or half-hourly sessions with a practitioner; additionally, group hypnosis interventions also exist. Some medical health specialists introduce hypnosis in 10-15-minute sessions along with their regular clinical work. In some cases, patients are taught to induce self-hypnosis (which is also accomplished by posthypnotic suggestion) [78].

Similarly to other techniques described here, hypnosis has been tried in many medical contexts. Effectiveness has been reported in irritable bowel syndrome [79], headache treatment $[80,81]$, chronic pain, and anxiety [82]. There is probable effectiveness of hypnotherapy as relief support in oncological and odontological procedures and promising outlook in interventions in diabetes and the human papilloma virus [83]. There are reports suggesting a positive role hypnosis can play for the functioning of the immune system [20]. The report developed in Evidence Synthesis Program 
TABLE 3: Health conditions for which hypnosis interventions had evidence of a positive effect on diagnosis-related, secondary, or global outcomes according to the U.S. Department of Veterans Affairs Evidence Synthesis Program (ESP) report published in 2019 [77].

\begin{tabular}{lccc}
\hline Condition & $\begin{array}{c}\text { Diagnosis-related } \\
\text { outcomes }\end{array}$ & $\begin{array}{c}\text { Secondary } \\
\text { outcomes }\end{array}$ & $\begin{array}{c}\text { Global } \\
\text { outcomes }\end{array}$ \\
\hline $\begin{array}{l}\text { Anxiety in cancer } \\
\text { patients }\end{array}$ & $\mathrm{X}$ & & \\
$\begin{array}{l}\text { Breast cancer } \\
\text { care }\end{array}$ & $\mathrm{X}$ & \\
$\begin{array}{l}\text { Obesity/weight } \\
\text { loss }\end{array}$ & $\mathrm{X}$ & \\
\hline
\end{tabular}

supported conclusion about evident positive effect of hypnotherapy in cancer-related care and obesity treatment. Probable positive effects were pointed in irritable bowel syndrome and reducing anxiety related to undergoing medical procedures [77] (see Table 3).

Some authors suggest hypnosis is underused in medical setting, considering its already known utility [84]. Application and effectiveness of hypnosis in medical setting depend on obeying fundamental rules, which encompass the need and willingness of the patient to get rid of the complaint, believe in the method, and trust in the practitioner applying it [85]. An important factor worth mentioning in the context of hypnotic treatment effectiveness is specific individual difference characteristic: susceptibility to hypnotic suggestion, referred to as hypnotic suggestibility (also known as hypnotizability or hypnotic susceptibility [86]). It is a trait relatively stable, measurable by means of several standard procedures, partially heritable [87]. Some authors suggested it to be a core variable in responsiveness to hypnotherapy in clinical setting [88]. Meta-analysis approach, however, exhibited only small and moderate effects of this trait in the mediation of hypnotic therapy effectiveness [86]. Research shows most medical patients regardless of hypnotic susceptibility may benefit from the integration of hypnotic therapies into their medical care, as so hypnotizability testing is rather recommended to detect cases of low susceptibility, who may benefit more from alternative approaches [87].

\section{Guided Imagery and Visualisations}

A precise definition of guided imagery has been stated by Rider and Achterberg [89] in their 1989 paper, where they describe it as "the internal experience of a perceptual event in the absence of the actual external stimuli." Guided imagery is thus invoking one or more senses experience with the use of imagination and guidance of specially designed instruction. Concentrating attention on envisioned images and scenarios reduces uncomfortable thoughts and feelings and distracts the mind from unwanted and negative state [90].

There are reports suggesting stress reduction effects and elevation of the immune system functioning in people treated with the guided imagery technique [23]. Additionally, pain relief effects had been observed [91]. In 2005, Roffe and col-
TABLE 4: Health conditions for which guided imagery interventions had evidence of a positive effect on diagnosis-related, secondary, or global outcomes according to the U.S. Department of Veterans Affairs Evidence Synthesis Program (ESP) report published in 2019 [77].

\begin{tabular}{lccc}
\hline Condition & $\begin{array}{c}\text { Diagnosis- } \\
\text { related outcomes }\end{array}$ & $\begin{array}{c}\text { Secondary } \\
\text { outcomes }\end{array}$ & $\begin{array}{c}\text { Global } \\
\text { outcomes }\end{array}$ \\
\hline $\begin{array}{l}\text { Arthritis/rheumatic } \\
\text { diseases }\end{array}$ & $\mathrm{X}$ & & \\
Cancer & & $\mathrm{X}$ & \\
\hline
\end{tabular}

leagues [92] in their studies researching guided imagery effectiveness in cancer patients concluded it as playing an adjuvant, psychosupportive, and comfort increasing role, but with no compelling evidence suggesting positive effects on physical symptoms.

The EVP report [93] notes a high level of confidence for usefulness of guided imagery techniques in arthritis/rheumatic disease and potential positive effects in interventions regarding Parkinson's disease, stroke, cancer, and menstrual disorders (see Table 4). A beneficial role has been also noted in supporting patients treated in Intensive Care Units (ICU) and persons undergoing cardiac surgery procedures.

\section{Relaxation Techniques}

Relaxation techniques are a large set of methods that are commonly being divided into two main categories: somatic(also called "physical") and cognitive- (also called "nonphysical") based approaches. The physical category includes for example pure behavioral relaxation training, progressive muscle relaxation, deep breathing, the Alexander technique and Feldenkrais method (education techniques aiming at establishing a heightened awareness of movements), and Mitchell's simple physiological relaxation [94-96]. Cognitive or nonphysical approaches include, among others, autogenic training - first described already in 1932 [97] — and Benson's method $[98,99]$ which are more focused on accomplishing the relaxation state by a person following the specially crafted instructions, rather than physical work with the body.

Relaxation techniques have been administered to support curing and treatment of various clinical conditions (see Table 5). Multiple relaxation methods are used as an adjuvant to treat pain. Progressive muscle relaxation (PMR) (combined with guided imagery) in cancer patients brought positive results in pain relief [100]. The Mitchell metho$\mathrm{d}$-administered for one month-managed to reduce symptoms accompanying fibromyalgia [101]. An older review of usefulness of relaxation techniques in acute pain management showed however poor performance of these methods [102]. Relaxation techniques proved to be useful in treatments of nausea of different origins, chemotherapy-caused $[103,104]$ or pregnancy-related [105]. Beneficiary effects have been reported in Chronic Obstructive Pulmonary Disease (COPD) (regarding both respiratory functions improvement and better psychological wellbeing) [106] and in ischaemic heart disease recovery efforts [107]. Relaxation 
TABLE 5: Health conditions treated with relaxation techniques with reported positive effect outcomes [101-107].

\begin{tabular}{lc}
\hline Condition & Method \\
\hline Cancer pain & $\begin{array}{c}\text { Progressive muscle } \\
\text { relaxation }\end{array}$ \\
Fibromyalgia & Mitchell method \\
Nausea-chemotherapy-caused & $\begin{array}{c}\text { Progressive muscle } \\
\text { relaxation }\end{array}$ \\
Nausea-pregnancy-related & Benson's method \\
Chronic Obstructive Pulmonary & Various relaxation \\
Disease & techniques \\
Ischaemic heart disease recovery & Various relaxation \\
techniques
\end{tabular}

techniques are commonly used as accompanying methods to alleviate symptoms and improve psychological health in illnesses, like edema, anxiety, and depression in postmastectomy lymphedema patients [108] or distress in people with diabetes [109].

\section{Disclosure Interventions}

The theoretical basis of the disclosure intervention methods lays in the supposition that inhibition of psychologically significant thoughts and feelings has adverse health consequences, $[19,110]$. In the 1980s, Pennebaker et al. in their correlational studies uncovered a pattern suggesting that not disclosing stressful experiences to others (e.g., spouse death, Holocaust survivorship, incest, and personal failures) is related to poorer health $[111,112]$. Pennebaker and Beall aimed later at reversing emotional suppression by developing an experimental paradigm in which individuals could disclose and process stressful experiences $[113,114]$.

The most commonly employed technique in this approach is "written emotional disclosure" or "expressive writing" that consists of 15 to 30-minute long sessions (often repeated in consecutive days) of writing essays on personally experienced stressful events. Sometimes instead of written, verbal descriptions are used [115], or the disclosure takes place by means of drawing or combination of writing and drawing [116].

The disclosure-based methods have been employed in many health problem contexts, exhibiting modest benefits in chronic pain (limited in rheumatoid arthritis, far better performance in fibromyalgia, mixed in headaches, cancer pain, pelvic pain, and abdominal pain) [113] and noticeable positive effects in wound healing $[117,118]$. Earlier, grander reviews in the form of meta-analysis produced mixed conclusions regarding disclosure interventions efficacy. Frisina and colleagues [119] reported significant positive improvements in health in clinical populations, with greater impact on physical than psychological health outcomes. In contrast, Meads and Nouwen concluded the nonexistence of emotional disclosure effects and suggested the reassessment of the method validity [120]. What appears to be of crucial importance in the context of disclosure effectiveness is the consideration of additional moderating factors such as emo- tional expressivity and baseline affect [121, 122], cognitiveemotional processing [123], social constraints [124], and ethnicity and form of the writing instruction [125].

\section{Meditation and Mindfulness Interventions}

Meditation is a practice in most general terms grounded in controlling focus of attention. It is referred in the classical languages of Buddhism-Sanskrit and Pali-as samādhi, etymologically meaning "gathering the mind and placing it upon an object" [126]. It can be categorized on the broadest level into three types. Concentration meditation depends on focusing attention on an object until quieting of the thoughts occurs in a subject's mind. Mindfulness meditation on the other hand emphasizes nonjudgmental openness to contents that arise in the mind spontaneously, not following and suspending reactions to them, promoting expanded awareness of present moment. Contemplative meditation is a mixture integrating both approaches, trying to combine focus with mind openness $[127,128]$.

Mindfulness meditation is the basis of mindfulness interventions. These interventions share the core premise of mindfulness - a process of openly attending, with awareness, to one's present moment experience-however referred by name to intervention schemes and programs [129].

A substantial body of research evidence, comprehensively reviewed by Sampaio and colleagues, cites important effects of meditation on not only body function parameters like reduction in respiratory frequency, slower heart rate, and reduced electrical conductivity of the skin [130] but also significant neuroendocrine and neurochemistry effects including structural changes to brain areas [131]. Other worth noticing positive influences on health and treatment that had been observed encompass a damping effect on $\mathrm{T}$ CD4+ lymphocyte reduction in adults infected with HIV-1, increased NK (Natural Killer) cytolytic activity [132-134], reduced risk for mortality in patients with coronary disease [135], and numerous evidence for enhancement in mood and well-being, decrease in stress, anxiety, and depression symptoms in healthy and ill individuals $[134,136,137]$.

\section{Discussion}

A short review of the most prominent groups of therapeutic methods in the field of behavioral medicine shows a large potential in the context of contemporary health care system use. One of the main advantages of the reviewed methods is their intrinsic noninvasive and pharmacology free essence. This characteristic positions them as promising alternatives for treating conditions in which pharmacological or other types of therapies are burdened with harmful risks. A good example here might be the problem of pain treatment. Currently, opioid analgesics are associated with one of the biggest US public health crises: the opioid crisis. Research showed that overwhelming majority of the opioid abusers began their addiction with prescription medications, primarily for chronic pain $[138,139]$. Pain, and particularly chronic pain, is the condition for which many of the reviewed behavioral medicine treatment methods proved to be efficacious, with 
a high level of confidence. Various biofeedback techniques, especially EMG, HRV, and thermal feedback, had been successfully administered in pain treatment-including chronic pain. Utility in this context has also been reported for relaxation techniques, with some more moderate evidence also found for hypnosis and guided imagery. Broader implementation of the best techniques from behavioral medicine repertoire at specific pain problems in clinical populations can bring relief to patients reducing the need of using medication, thus lessening the risks and potential of negative consequences associated with painkiller drugs. It is worth mentioning that usage of alternative, nonpharmacological treatments of pain was much more common in years before the emergence of opioid crisis [140].

Another distinctive advantage of behavioral medicine treatments is the fact that many of them are founded on learning processes and rely on training. This provides possibilities unavailable to other therapies, like surgery or pharmacotherapy. Persons treated with the learning-based therapies acquire skills and knowledge, meaning that in very many cases (those not requiring specialized apparatus like fMRI feedback), they can apply the treatment to themselves whenever necessary on their own. Furthermore, they keep the trained skill and can use them long after they acquired it and also possibly apply it outside the original illness context. Relaxation, expanding mindfulness, and self-hypnosis are skills which can beneficially be applied in health and even outside of health related context, for the rest of life. This means contact with some of the behavioral medicine techniques means arming people with useful techniques they can apply for promoting their health and improve wellbeing in the long term. This in turn might equal more years lived in better health and less strain on health care system.

Another leverage associated with many of the reviewed methods is their big potential in the context of convenient, remote, offsite use (eHealth) [141, 142]. Used case examples have already been reported for mindfulness, relaxation techniques, and hypnosis administered via tools such as smartphone applications or tele-sessions in which the therapist uses video call capabilities to remotely conduct intervention with a patient $[129,141,143]$. Automation and saving potential are clearly visible here.

Behavioral medicine-derived medical treatment field is also affected with problems. The issue repeatedly mentioned in the literature is deficiency of high-quality research that would enable drawing definitive conclusions regarding the effectiveness of many of the considered methods [71, 93]. Till now, only minority out of plentitude of mentioned here interventions had been conclusively confirmed or rejected as useful therapies in given conditions. Until effectiveness will be established with high-quality evidence, the dissemination of behavioral medicine therapies in medical setting will understandably not be high. Another major problem, along insufficiency of effectiveness evidence in many of the use cases, is similar deficit of empirically sound explanations of mechanisms underlying therapeutic benefits. Solving these two major deficiencies is a key prerequisite for enhancing the use of behavioral medicine treatment approaches in clinical setting.

\section{Conclusions}

A behavioral medicine framework provides wealth of methods for use in the treatment of multitude somatic health conditions. Those alternative approaches hold substantial potential in terms of reducing risks and unwanted negative consequences in cases where they can effectively substitute pharmacological or invasive therapies. Another useful area of employment for these methods is complementary and supporting role along the main treatment. Broader dissemination and popularization of these techniques can occur when insufficiencies regarding evidence of effectiveness and underlying curing mechanisms will be tackled.

\section{Conflicts of Interest}

The authors declare no conflict of interest.

\section{Authors' Contributions}

$\mathrm{JB}, \mathrm{JJ}$, and JW are responsible for the conceptualization, writing and original draft preparation, review, and editing. All authors have read and agreed to the published version of the manuscript.

\section{Acknowledgments}

This study was supported by statutory grant No. 61610001100 , from the University of Warmia and Mazury in Olsztyn, Poland.

\section{References}

[1] H. G. Koenig and H. J. Cohen, The Link between Religion and Health: Psychoneuroimmunology and the Faith Factor, Oxford University Press, 2002.

[2] N. Deuraseh and M. Abu Talib, "Mental health in Islamic medical tradition," International Medical Journal, vol. 4, no. 2, pp. 76-79, 2005.

[3] B. B. Raginsky, "Psychosomatic medicine," The American Journal of Medicine, vol. 5, no. 6, pp. 857-878, 1948.

[4] G. E. Schwartz and S. M. Weiss, "Behavioral medicine revisited: an amended definition," Journal of Behavioral Medicine, vol. 1, no. 3, pp. 249-251, 1978.

[5] G. E. Schwartz, "Testing the biopsychosocial model: The ultimate challenge facing behavioral medicine?," Journal of Consulting and Clinical Psychology, vol. 50, no. 6, pp. 10401053, 1982.

[6] E. B. Fisher et al., Principles and Concepts of Behavioral Medicine: A Global Handbook, Springer, 2018.

[7] The Intearnational Society of Behavioral Medicine, "Charter,” March 2020, https://www.isbm.info/about-isbm/ charter/.

[8] J. Dekker, A. Stauder, and F. J. Penedo, "Proposal for an update of the definition and scope of behavioral medicine," International Journal of Behavioral Medicine, vol. 24, no. 1, pp. 1-4, 2017.

[9] S. M. Weiss and G. E. Schwartz, "Behavioral medicine: a retro/prospective view of the field," Journal of Behavioral Medicine, vol. 42, no. 1, pp. 5-11, 2019. 
[10] R. Ader, "Psychoneuroimmunology," in International Encyclopedia of the Social \& Behavioral Sciences, N. J. Smelser and P. B. Baltes, Eds., pp. 12422-12428, Pergamon, Oxford, 2001.

[11] F. R. Brush and S. Levine, Psychoendocrinology, Academic Press, 2013.

[12] W. S. Breitbart and Y. Alici, "Psycho-oncology," Harvard Review of Psychiatry, vol. 17, no. 6, pp. 361-376, 2009.

[13] T. A. Revenson and R. A. R. Gurung, Handbook of Health Psychology, Routledge, 2018.

[14] R. Zachariae, "Psychoneuroimmunology: a bio-psycho-social approach to health and disease," Scandinavian Journal of Psychology, vol. 50, no. 6, pp. 645-651, 2009.

[15] D. K. Wilson, A. Christensen, P. B. Jacobsen, and R. M. Kaplan, "Standards for economic analyses of interventions for the field of health psychology and behavioral medicine," Health Psychology, vol. 38, no. 8, pp. 669-671, 2019.

[16] G. E. Schwartz and S. M. Weiss, "What is Behavioral Medicine," Psychosomatic Medicine, vol. 39, no. 6, pp. 377-381, 1977.

[17] D. L. Frank, L. Khorshid, J. F. Kiffer, C. S. Moravec, and M. G. McKee, "Biofeedback in medicine: who, when, why and how?," Mental Health in Family Medicine, vol. 7, no. 2, pp. 85-91, 2010.

[18] A.-L. Lumma, B. E. Kok, and T. Singer, "Is meditation always relaxing? Investigating heart rate, heart rate variability, experienced effort and likeability during training of three types of meditation," International Journal of Psychophysiology, vol. 97, no. 1, pp. 38-45, 2015.

[19] G. E. Miller and S. Cohen, "Psychological interventions and the immune system: a meta-analytic review and critique," Health Psychology, vol. 20, no. 1, pp. 47-63, 2001.

[20] P. Neumann, "The use of hypnosis in modifying immune system response," Australian Journal of Clinical and Experimental Hypnosis, vol. 33, no. 2, p. 140, 2005.

[21] B. S. Peavey, G. F. Lawlis, and A. Goven, "Biofeedbackassisted relaxation: effects on phagocytic capacity," Biofeedback and Self-Regulation, vol. 10, no. 1, pp. 33-47, 1985.

[22] P. Sedlmeier, J. Eberth, M. Schwarz et al., "The psychological effects of meditation: a meta-analysis," Psychological Bulletin, vol. 138, no. 6, pp. 1139-1171, 2012.

[23] E. C. Trakhtenberg, "The effects of guided imagery on the immune system: a critical review," The International Journal of Neuroscience, vol. 118, no. 6, pp. 839-855, 2009.

[24] L. Varvogli and C. Darviri, "Stress management techniques: evidence-based procedures that reduce stress and promote health," Health Science Journal, vol. 5, no. 2, p. 74, 2011.

[25] C. Yucha and D. Montgomery, Evidence-based practice in biofeedback and neurofeedback, 2008, https:// digitalscholarship.unlv.edu/nursing_fac_articles/1.

[26] M. A. C. García and T. M. M. Vieira, "Surface electromyography: why, when and how to use it," Revista Andaluza de Medicina del Deporte, vol. 4, no. 1, pp. 17-28, 2011.

[27] R. Neblett, "Surface electromyographic (SEMG) biofeedback for chronic low back pain," Healthcare, vol. 4, no. 2, p. 27, 2016.

[28] E. Jacobson, "Electrical measurements of neuromuscular states during mental activities," American Journal of Physiology-Legacy Content, vol. 95, no. 3, pp. 703-712, 1930.

[29] E. Jacobson, "Electrical measurements concerning muscular contraction (tonus) and the cultivation of relaxation in man-relaxation-times of individuals," American Journal of Physiology-Legacy Content, vol. 108, no. 3, pp. 573-580, 1934.

[30] J. A. Astin, W. Beckner, K. Soeken, M. C. Hochberg, and B. Berman, "Psychological interventions for rheumatoid arthritis: a meta-analysis of randomized controlled trials," Arthritis Care and Research, vol. 47, no. 3, pp. 291-302, 2002.

[31] A. Huntley, A. R. White, and E. Ernst, "Relaxation therapies for asthma: a systematic review," Thorax, vol. 57 , no. 2 , pp. 127-131, 2002.

[32] E. Dalla Toffola, D. Bossi, M. Buonocore, C. Montomoli, L. Petrucci, and E. Alfonsi, "Usefulness of BFB/EMG in facial palsy rehabilitation," Disability and Rehabilitation, vol. 27, no. 14, pp. 809-815, 2009.

[33] J. E. Bolek, "What physicians need to know about surface electromyography," Biofeedback, vol. 45, no. 1, pp. 9-11, 2017.

[34] A. MacIntosh, E. Lam, V. Vigneron, N. Vignais, and E. Biddiss, "Biofeedback interventions for individuals with cerebral palsy: a systematic review," Disability and Rehabilitation, vol. 41, no. 20, pp. 2369-2391, 2019.

[35] K. J. Lizarraga, D. Al-Shorafat, and S. Fox, "Update on current and emerging therapies for dystonia," Neurodegenerative Disease Management, vol. 9, no. 3, pp. 135-147, 2019.

[36] A. Albanese, M. Di Giovanni, and S. Lalli, "Dystonia: diagnosis and management," European Journal of Neurology, vol. 26, no. 1, pp. 5-17, 2018.

[37] E. Peper, K. H. Gibney, and V. E. Wilson, "Group training with healthy computing practices to prevent repetitive strain injury (RSI): a preliminary study," Applied Psychophysiology and Biofeedback, vol. 29, no. 4, pp. 279-287, 2004.

[38] S. Cotterill, Performance Psychology: Theory and Practice, Routledge, 2017.

[39] Y. Nestoriuc, A. Martin, W. Rief, and F. Andrasik, "Biofeedback treatment for headache disorders: a comprehensive efficacy review," Applied Psychophysiology and Biofeedback, vol. 33, no. 3, pp. 125-140, 2008.

[40] I. Khazan, "Biofeedback treatment for headaches," Biofeedback, vol. 45, no. 2, pp. 34-35, 2017.

[41] A. Crider, A. G. Glaros, and R. N. Gevirtz, "Efficacy of biofeedback-based treatments for temporomandibular disorders," Applied Psychophysiology and Biofeedback, vol. 30, no. 4, pp. 333-345, 2005.

[42] S. Heymen, Y. Scarlett, K. Jones, Y. Ringel, D. Drossman, and W. E. Whitehead, "Randomized, controlled trial shows biofeedback to be superior to alternative treatments for patients with pelvic floor dyssynergia-type constipation," Diseases of the Colon and Rectum, vol. 50, no. 4, pp. 428-441, 2007.

[43] M. K. Karavidas, P.-S. Tsai, C. Yucha, A. McGrady, and P. M. Lehrer, "Thermal biofeedback for primary Raynaud's phenomenon: a review of the literature," Applied Psychophysiology and Biofeedback, vol. 31, no. 3, pp. 203-216, 2006.

[44] R. Kaushik, R. M. Kaushik, S. K. Mahajan, and V. Rajesh, "Biofeedback assisted diaphragmatic breathing and systematic relaxation versus propranolol in long term prophylaxis of migraine," Complementary Therapies in Medicine, vol. 13, no. 3, pp. 165-174, 2005.

[45] P. A. Humphreys and R. N. Gevirtz, "Treatment of recurrent abdominal pain: components analysis of four treatment protocols," Journal of Pediatric Gastroenterology and Nutrition, vol. 31, no. 1, pp. 47-51, 2000. 
[46] M. S. Schwartz and F. Andrasik, Biofeedback, Fourth Edition, A Practitioner's Guide. Guilford Publications, 2017.

[47] J.-A. Micoulaud-Franchi, I. Kotwas, L. Lanteaume et al., "Skin conductance biofeedback training in adults with drugresistant temporal lobe epilepsy and stress-triggered seizures: a proof-of-concept study," Epilepsy \& Behavior, vol. 41, pp. 244-250, 2014.

[48] Y. Nagai, "Autonomic biofeedback therapy in epilepsy," Epilepsy Research, vol. 153, pp. 76-78, 2019.

[49] Y. Nagai, C. I. Jones, and A. Sen, "Galvanic skin response (gsr)/electrodermal/skin conductance biofeedback on epilepsy: a systematic review and meta-analysis," Frontiers in Neurology, vol. 10, p. 377, 2019.

[50] R. D. Sheth, C. E. Stafstrom, and D. Hsu, "Nonpharmacological treatment options for epilepsy," Seminars in Pediatric Neurology, vol. 12, no. 2, pp. 106-113, 2005.

[51] M. Kumar, D. Pandey, and P. Shrivastva, "Effect of GSR biofeedback relaxation training on blood glucose and anxiety level of type 2 diabetic patients," International Journal of Indian Psychology, vol. 4, no. 1, 2016.

[52] Y. Nagai, "Modulation of autonomic activity in neurological conditions: epilepsy and Tourette syndrome," Frontiers in Neuroscience, vol. 9, 2015.

[53] H. J. Lee, J. H. Lee, E. Y. Cho, S. M. Kim, and S. Yoon, "Efficacy of psychological treatment for headache disorder: a systematic review and meta-analysis," The Journal of Headache and Pain, vol. 20, no. 1, p. 17, 2019.

[54] P. M. Lehrer and R. Gevirtz, "Heart rate variability biofeedback: how and why does it work?," Frontiers in Psychology, vol. 5, 2014.

[55] P. Windthorst, N. Mazurak, M. Kuske et al., "Heart rate variability biofeedback therapy and graded exercise training in management of chronic fatigue syndrome: an exploratory pilot study," Journal of Psychosomatic Research, vol. 93, pp. 6-13, 2017.

[56] K. S. Masters, "Recurrent abdominal pain, medical intervention, and biofeedback: what happened to the biopsychosocial model?," Applied Psychophysiology and Biofeedback, vol. 31, no. 2, pp. 155-165, 2006.

[57] R. Gevirtz, "The muscle spindle trigger point model of chronic pain," Biofeedback, vol. 34, no. 2, p. 53, 2006.

[58] B. Slutsker, A. Konichezky, and D. Gothelf, "Breaking the cycle: cognitive behavioral therapy and biofeedback training in a case of cyclic vomiting syndrome," Psychology, Health \& Medicine, vol. 15, no. 6, pp. 625-631, 2010.

[59] T. Renton, A. Tibbles, and J. Topolovec-Vranic, "Neurofeedback as a form of cognitive rehabilitation therapy following stroke: a systematic review," PLoS One, vol. 12, no. 5, article e0177290, 2017.

[60] N. Omejc, B. Rojc, P. P. Battaglini, and U. Marusic, "Review of the therapeutic neurofeedback method using electroencephalography: EEG neurofeedback," Bosnian Journal of Basic Medical Sciences, vol. 19, no. 3, pp. 213-220, 2019.

[61] E. Weber, A. Köberl, S. Frank, and M. Doppelmayr, "Predicting successful learning of SMR neurofeedback in healthy participants: methodological considerations," Applied Psychophysiology and Biofeedback, vol. 36, no. 1, pp. 37-45, 2011.

[62] J. E. Walker and G. P. Kozlowski, "Neurofeedback treatment of epilepsy," Child and Adolescent Psychiatric Clinics of North America, vol. 14, no. 1, pp. 163-176, 2005.
[63] S. Kayiran, E. Dursun, N. Dursun, N. Ermutlu, and S. Karamürsel, "Neurofeedback intervention in fibromyalgia syndrome; a randomized, controlled, rater blind clinical trial," Applied Psychophysiology and Biofeedback, vol. 35, no. 4, pp. 293-302, 2010.

[64] S. Chen, J. Li, S. Li, L. Qian, Y. He, and B. Liu, "Biofeedback therapy for chronic prostatitis: application and consideration," Zhonghua nan ke xue= National Journal of Andrology, vol. 22, no. 1, pp. 57-62, 2016.

[65] J. Białkowska, D. Mroczkowska, J. Wojtkiewicz, and W. Maksymowicz, "The use of EEG biofeedback in rehabilitation of a patient with amyotrophic lateral sclerosis with cognitive, mood and motivation disorders," Polish Annals of Medicine, vol. 25, no. 1, pp. 68-73, 2018.

[66] A. Mottaz, M. Solcà, C. Magnin, T. Corbet, A. Schnider, and A. G. Guggisberg, "Neurofeedback training of alpha-band coherence enhances motor performance," Clinical Neurophysiology, vol. 126, no. 9, pp. 1754-1760, 2015.

[67] E. López-Larraz, C. Escolano, L. Montesano, and J. Minguez, "Reactivating the dormant motor cortex after spinal cord injury with EEG neurofeedback: a case study with a chronic, complete C4 patient," Clinical EEG and Neuroscience, vol. 50, no. 2, article 1550059418792153, pp. 100-110, 2018.

[68] R. Rostami, P. Salamati, K. K. Yarandi et al., "Effects of neurofeedback on the short-term memory and continuous attention of patients with moderate traumatic brain injury: a preliminary randomized controlled clinical trial," Chinese Journal of Traumatology, vol. 20, no. 5, pp. 278-282, 2017.

[69] D. C. Hammond, "Can LENS neurofeedback treat anosmia resulting from a head injury?," Journal of Neurotherapy, vol. 11, no. 1, pp. 57-62, 2007.

[70] R. T. Thibault and A. Raz, "When can neurofeedback join the clinical armamentarium?," Lancet Psychiatry, vol. 3, no. 6, pp. 497-498, 2016.

[71] R. T. Thibault, M. Lifshitz, and A. Raz, "Neurofeedback or neuroplacebo?," Brain, vol. 140, no. 4, pp. 862-864, 2017.

[72] R. Sitaram, T. Ros, L. Stoeckel et al., "Closed-loop brain training: the science of neurofeedback," Nature Reviews Neuroscience, vol. 18, no. 2, pp. 86-100, 2017.

[73] R. T. Thibault, A. MacPherson, M. Lifshitz, R. R. Roth, and A. Raz, "Neurofeedback with fMRI: a critical systematic review," NeuroImage, vol. 172, pp. 786-807, 2018.

[74] T. Watanabe, Y. Sasaki, K. Shibata, and M. Kawato, "Advances in fMRI real-time neurofeedback," Trends in Cognitive Sciences, vol. 21, no. 12, pp. 997-1010, 2017.

[75] L. E. Stoeckel, K. A. Garrison, S. S. Ghosh et al., "Optimizing real time fMRI neurofeedback for therapeutic discovery and development," NeuroImage: Clinical, vol. 5, pp. 245-255, 2014.

[76] D. Moss and J. Gunkelman, "Task force report on methodology and empirically supported treatments: introduction," Applied Psychophysiology and Biofeedback, vol. 27, no. 4, pp. 271-272, 2002.

[77] M. Freeman, C. Ayers, K. Kondo et al., Guided imagery, biofeedback, and hypnosis: a map of the evidence, VA ESP Proj., 2019.

[78] A. Vickers, C. Zollman, and D. K. Payne, "Hypnosis and relaxation therapies," The Western Journal of Medicine, vol. 175, no. 4, pp. 269-272, 2001.

[79] C. E. Flik, W. Laan, N. P. A. Zuithoff et al., "Efficacy of individual and group hypnotherapy in irritable bowel syndrome 
(IMAGINE): a multicentre randomised controlled trial," The Lancet Gastroenterology \& Hepatology, vol. 4, no. 1, pp. 2031, 2019.

[80] A. M. Ahmadi, M. Jafari, L. Sabzevari, A. FallahTafti, and R. Bidaki, "Evaluation of the effect of hypnotherapy on the headache," Sleep and Hypnosis, vol. 20, no. 2, pp. 114-119, 2017.

[81] N. Flynn, "Systematic review of the effectiveness of hypnosis for the management of headache," The International Journal of Clinical and Experimental Hypnosis, vol. 66, no. 4, pp. 343352, 2018.

[82] E. Davis, "Literature review of the evidence-base for the effectiveness of hypnotherapy," PACFA, Psychotheraphy \& Counselling Federation of Australia, Melbourne, 2015.

[83] J. A. Molina del Peral, M. E. Mendoza, L. Díez González, Y. Llanes Basulto, and A. Capafons, "Clinical hypnosis: assessment of its effectiveness and the quality of the investigation of it in medicine," Revista del Hospital Psiquiátrico de La Habana, vol. 13, no. 3, 2017.

[84] V. M. Yeh, J. B. Schnur, and G. H. Montgomery, "Disseminating hypnosis to health care settings: applying the REAIM framework," Psychology of Consciousness: Theory, Research and Practice, vol. 1, no. 2, pp. 213-228, 2014.

[85] Ö. Erel and K. V. Erel, "Hypnosis," Meandros Medical and Dental Journal, vol. 18, no. 2, pp. 76-79, 2017.

[86] G. H. Montgomery, J. B. Schnur, and D. David, "The impact of hypnotic suggestibility in clinical care settings," The International Journal of Clinical and Experimental Hypnosis, vol. 59, no. 3, pp. 294-309, 2011.

[87] D. Moss and E. Willmarth, "Hypnosis, anesthesia, pain management, and preparation for medical procedures," Annals of Palliative Medicine, vol. 8, no. 4, pp. 498-503, 2019.

[88] A. Barabasz and N. Perez, "Salient findings: hypnotizability as core construct and the clinical utility of hypnosis," International Journal of Clinical and Experimental Hypnosis, vol. 55, no. 3, pp. 372-379, 2007.

[89] M. S. Rider and J. Achterberg, "Effect of music-assisted imagery on neutrophils and lymphocytes," Biofeedback and SelfRegulation, vol. 14, no. 3, pp. 247-257, 1989.

[90] J. de Leyer-Tiarks, L. Gammie, A. M. Bray, and S. Moriarty, Relaxation and guided imagery for mind-body health, pp. 173-186, American Psychological Association, 2020.

[91] K. Bernardy, N. Füber, P. Klose, and W. Häuser, "Efficacy of hypnosis/guided imagery in fibromyalgia syndrome - a systematic review and meta-analysis of controlled trials," BMC Musculoskeletal Disorders, vol. 12, no. 1, p. 133, 2011.

[92] L. Roffe, K. Schmidt, and E. Ernst, "A systematic review of guided imagery as an adjuvant cancer therapy," PsychoOncology, vol. 14, no. 8, pp. 607-617, 2005.

[93] K. Kondo, K. M. Noonan, M. Freeman, C. Ayers, B. J. Morasco, and D. Kansagara, "Efficacy of biofeedback for medical conditions: an evidence map," Journal of General Internal Medicine, vol. 34, no. 12, pp. 2883-2893, 2019.

[94] S. Jain, K. Janssen, and S. DeCelle, "Alexander technique and Feldenkrais method: a critical overview," Physical Medicine and Rehabilitation Clinics of North America, vol. 15, no. 4, pp. 811-825, 2004.

[95] K. Kerr, "Critical review on relaxation techniques," Critical Reviews in Physical and Rehabilitation Medicine, vol. 29, no. 1-4, pp. 390-428, 2017.
[96] J. Whittingham, "Relaxation techniques - a practical handbook for the health care professional," Physiotherapy, vol. 82 , no. 6, p. 378, 1996.

[97] F. Stetter and S. Kupper, "Autogenic training: a meta-analysis of clinical outcome studies," Applied Psychophysiology and Biofeedback, vol. 27, no. 1, pp. 45-98, 2002.

[98] J. F. Beary, H. Benson, and H. P. Klemchuk, "A simple psychophysiologic technique which elicits the hypometabolic changes of the relaxation response," Psychosomatic Medicine, vol. 36, no. 2, pp. 115-120, 1974.

[99] M. Rambod, N. Pourali-Mohammadi, N. Pasyar, F. Rafii, and F. Sharif, "The effect of Benson's relaxation technique on the quality of sleep of Iranian hemodialysis patients: a randomized trial," Complementary Therapies in Medicine, vol. 21, no. 6, pp. 577-584, 2013.

[100] G. de Paolis, A. Naccarato, F. Cibelli et al., "The effectiveness of progressive muscle relaxation and interactive guided imagery as a pain-reducing intervention in advanced cancer patients: a multicentre randomised controlled nonpharmacological trial," Complementary Therapies in Clinical Practice, vol. 34, pp. 280-287, 2019.

[101] A. Amirova, M. Cropley, and A. Theadom, "The effectiveness of the Mitchell Method Relaxation Technique for the treatment of fibromyalgia symptoms: a three-arm randomized controlled trial," International Journal of Stress Management, vol. 24, no. 1, pp. 86-106, 2017.

[102] K. Seers and D. Carroll, "Relaxation techniques for acute pain management: a systematic review," Journal of Advanced Nursing, vol. 27, no. 3, pp. 466-475, 1998.

[103] I. Mastenbroek and L. McGovern, "The effectiveness of relaxation techniques in controlling chemotherapy induced nausea: a literature review," Australian Occupational Therapy Journal, vol. 38, no. 3, pp. 137-142, 1991.

[104] A. Molassiotis, H. P. Yung, B. M. Yam, F. Y. Chan, and T. Mok, "The effectiveness of progressive muscle relaxation training in managing chemotherapy-induced nausea and vomiting in Chinese breast cancer patients: a randomised controlled trial," Supportive Care in Cancer, vol. 10, no. 3, pp. 237-246, 2002.

[105] B. Fateme, M. K. Fatemeh, M. Vahid, N. J. Arezou, N. Manizhe, and M. Zahra, "The effect of Benson's muscle relaxation technique on severity of pregnancy nausea," Electronic Journal of General Medicine, vol. 16, no. 2, article em125, 2019.

[106] E. Volpato, P. Banfi, S. M. Rogers, and F. Pagnini, “Relaxation techniques for people with chronic obstructive pulmonary disease: a systematic review and a meta-analysis," EvidenceBased Complementary and Alternative Medicine, vol. 2015, Article ID 628365, 22 pages, 2015.

[107] J. van Dixhoorn and A. White, "Relaxation therapy for rehabilitation and prevention in ischaemic heart disease: a systematic review and meta-analysis," European Journal of Cardiovascular Prevention and Rehabilitation, vol. 12, no. 3, pp. 193-202, 2005.

[108] B. Abbasi, N. Mirzakhany, L. Angooti Oshnari et al., "The effect of relaxation techniques on edema, anxiety and depression in post-mastectomy lymphedema patients undergoing comprehensive decongestive therapy: a clinical trial," PloS One, vol. 13, no. 1, article e0190231, 2018.

[109] D. Raveendranathan, J. George, N. L. Perumal, and A. Mysore, "The effectiveness of a brief psychological intervention for patients with diabetes-related distress," Indian 
Journal of Psychological Medicine, vol. 41, no. 4, pp. 357-361, 2019.

[110] J. W. Pennebaker, "Emotion, disclosure, and health: an overview," in Emotion, Disclosure, \& Health, pp. 3-10, American Psychological Association, Washington, DC, USA, 1995.

[111] J. W. Pennebaker, S. D. Barger, and J. Tiebout, "Disclosure of traumas and health among holocaust survivors," Psychosomatic Medicine, vol. 51, no. 5, pp. 577-589, 1989.

[112] J. W. Pennebaker and R. C. O'Heeron, "Confiding in others and illness rate among spouses of suicide and accidentaldeath victims," Journal of Abnormal Psychology, vol. 93, no. 4, pp. 473-476, 1984.

[113] M. A. Lumley, E. R. Sklar, and J. N. Carty, "Emotional disclosure interventions for chronic pain: from the laboratory to the clinic," Translational Behavioral Medicine, vol. 2, no. 1, pp. 73-81, 2012.

[114] J. W. Pennebaker and S. K. Beall, "Confronting a traumatic event: toward an understanding of inhibition and disease," Journal of Abnormal Psychology, vol. 95, no. 3, pp. 274-281, 1986.

[115] B. A. Esterling, M. H. Antoni, M. A. Fletcher, S. Margulies, and N. Schneiderman, "Emotional disclosure through writing or speaking modulates latent Epstein-Barr virus antibody titers," Journal of Consulting and Clinical Psychology, vol. 62, no. 1, pp. 130-140, 1994.

[116] T. Pantchenko, M. Lawson, and M. R. Joyce, "Verbal and non-verbal disclosure of recalled negative experiences: relation to well-being," Psychology and Psychotherapy: Theory, Research and Practice, vol. 76, no. 3, pp. 251265, 2003.

[117] H. Robinson, The Impact of Psychological Interventions on Wound Healing, Thesis, ResearchSpace@Auckland, 2017.

[118] J. Weinman, M. Ebrecht, S. Scott, J. Walburn, and M. Dyson, "Enhanced wound healing after emotional disclosure intervention," British Journal of Health Psychology, vol. 13, no. 1, pp. 95-102, 2008.

[119] P. G. Frisina, J. C. Borod, and S. J. Lepore, “A meta-analysis of the effects of written emotional disclosure on the health outcomes of clinical populations," The Journal of Nervous and Mental Disease, vol. 192, no. 9, pp. 629-634, 2004.

[120] C. Meads and A. Nouwen, "Does emotional disclosure have any effects? A systematic review of the literature with metaanalyses," International Journal of Technology Assessment in Health Care, vol. 21, no. 2, pp. 153-164, 2005.

[121] A. N. Niles, K. E. B. Haltom, C. M. Mulvenna, M. D. Lieberman, and A. L. Stanton, "Randomized controlled trial of expressive writing for psychological and physical health: the moderating role of emotional expressivity," Anxiety, Stress, and Coping, vol. 27, no. 1, pp. 1-17, 2013.

[122] S. A. Norman, M. A. Lumley, J. A. Dooley, and M. P. Diamond, "For whom does it work? Moderators of the effects of written emotional disclosure in a randomized trial among women with chronic pelvic pain," Psychosomatic Medicine, vol. 66, no. 2, pp. 174-183, 2004.

[123] H. van Middendorp and R. Geenen, "Poor cognitiveemotional processing may impede the outcome of emotional disclosure interventions," British Journal of Health Psychology, vol. 13, no. 1, pp. 49-52, 2008.

[124] E. L. Merz, R. S. Fox, and V. L. Malcarne, "Expressive writing interventions in cancer patients: a systematic review," Health Psychology Review, vol. 8, no. 3, pp. 339-361, 2013.
[125] Q. Lu and A. L. Stanton, "How benefits of expressive writing vary as a function of writing instructions, ethnicity and ambivalence over emotional expression," Psychology \& Health, vol. 25, no. 6, pp. 669-684, 2010.

[126] A. Olendzki, "Mindfulness and meditation," in Clinical handbook of mindfulness, pp. 37-44, Springer, 2009.

[127] A. Lutz, H. A. Slagter, J. D. Dunne, and R. J. Davidson, "Attention regulation and monitoring in meditation," Trends in Cognitive Sciences, vol. 12, no. 4, pp. 163-169, 2008.

[128] C. V. S. Sampaio, M. G. Lima, and A. M. Ladeia, "Meditation, health and scientific investigations: review of the literature," Journal of Religion and Health, vol. 56, no. 2, pp. 411-427, 2017.

[129] J. D. Creswell, "Mindfulness interventions," Annual Review of Psychology, vol. 68, no. 1, pp. 491-516, 2017.

[130] Y.-Y. Tang, Y. Ma, Y. Fan et al., "Central and autonomic nervous system interaction is altered by short-term meditation," Proceedings of the National Academy of Sciences of the United States of America, vol. 106, no. 22, pp. 8865-8870, 2009.

[131] B. K. Hölzel, J. Carmody, M. Vangel et al., "Mindfulness practice leads to increases in regional brain gray matter density," Psychiatry Research, vol. 191, no. 1, pp. 36-43, 2011.

[132] J. D. Creswell, H. F. Myers, S. W. Cole, and M. R. Irwin, "Mindfulness meditation training effects on CD4+ T lymphocytes in HIV-1 infected adults: a small randomized controlled trial," Brain, Behavior, and Immunity, vol. 23, no. 2, pp. 184-188, 2009.

[133] C. Y. Fang, D. K. Reibel, M. L. Longacre, S. Rosenzweig, D. E. Campbell, and S. D. Douglas, "Enhanced psychosocial wellbeing following participation in a mindfulness-based stress reduction program is associated with increased natural killer cell activity," Journal of Alternative and Complementary Medicine, vol. 16, no. 5, pp. 531-538, 2010.

[134] L. Witek-Janusek, K. Albuquerque, K. R. Chroniak, C. Chroniak, R. Durazo-Arvizu, and H. L. Mathews, "Effect of mindfulness based stress reduction on immune function, quality of life and coping in women newly diagnosed with early stage breast cancer," Brain, Behavior, and Immunity, vol. 22, no. 6, pp. 969-981, 2008.

[135] R. H. Schneider, C. E. Grim, M. V. Rainforth et al., "Stress reduction in the secondary prevention of cardiovascular disease," Circulation: Cardiovascular Quality and Outcomes, vol. 5, no. 6, pp. 750-758, 2012.

[136] C. R. Gross, M. J. Kreitzer, W. Thomas et al., "Mindfulnessbased stress reduction for solid organ transplant recipients: a randomized controlled trial," Alternative Therapies in Health and Medicine, vol. 16, no. 5, pp. 30-38, 2010.

[137] C. J. Hoffman, S. J. Ersser, J. B. Hopkinson, P. G. Nicholls, J. E. Harrington, and P. W. Thomas, "Effectiveness of mindfulness-based stress reduction in mood, breast- and endocrine-related quality of life, and well-being in stage 0 to III breast cancer: a randomized, controlled trial," Journal of Clinical Oncology, vol. 30, no. 12, pp. 1335-1342, 2012.

[138] N. Vadivelu, A. M. Kai, V. Kodumudi, J. Sramcik, and A. D. Kaye, "The opioid crisis: a comprehensive overview," Current Pain and Headache Reports, vol. 22, no. 3, p. 16, 2018.

[139] M. A. Makary, H. N. Overton, and P. Wang, "Overprescribing Is Major Contributor to Opioid Crisis," British Medical Journal, vol. 359, article j4792, 2017. 
[140] N. Dasgupta, L. Beletsky, and D. Ciccarone, "Opioid crisis: no easy fix to its social and economic determinants," American Journal of Public Health, vol. 108, no. 2, pp. 182-186, 2018.

[141] M. Mikolasek, J. Berg, C. M. Witt, and J. Barth, "Effectiveness of mindfulness- and relaxation-based eHealth interventions for patients with medical conditions: a systematic review and synthesis," International Journal of Behavioral Medicine, vol. 25, no. 1, pp. 1-16, 2018.

[142] World Health Organization, eHealthMarch 2020, https:// www.who.int/ehealth/about/en/.

[143] S. S. Hasan, J. S. Pearson, J. Morris, and P. J. Whorwell, "Skype hypnotherapy for irritable bowel syndrome: effectiveness and comparison with face-to-face treatment," The International Journal of Clinical and Experimental Hypnosis, vol. 67, no. 1, pp. 69-80, 2019. 